Mikel Izquierdo

\title{
Biomecánica y Bases Neuromusculares de la Actividad Física y el Deporte
}

Editorial Médica Panamericana. 2008. ISBN: 9788498350234

\section{José Manuel Palao Andrés}

Facultad de Ciencias de la Salud, de la Actividad Física y del Deporte. Universidad Católica San Antonio, Murcia

\author{
CORRESPONDENCIA: \\ Universidad Católica San Antonio de Murcia \\ Departamento de Ciencias de la Actividad Física y del Deporte \\ Campus de los Jerónimos, s/n \\ 30107 Guadalupe (Murcia) \\ jmpalao@pdi.ucam.edu
}

Recepción: febrero 2010 • Aceptación: febrero 2010

- 1 presente libro aborda una de las temáticas básicas que forman parte del conocimiento en Ciencias de la Actividad Física y del Deporte. Los objetivos del mismo son presentar las bases para la comprensión y el estudio de cómo el movimiento humano y deportivo se produce a nivel mecánico y cómo éste afecta al cuerpo humano, todo ello siempre desde la perspectiva científica. Quizás no es correcto utilizar el término libro para hacer referencia al mismo, ya que el presente "manual de referencia" está compuesto por 37 capítulos independientes que no obstante encajan dentro del mismo como piezas de un puzle. Las 769 páginas de este manual están escritas por 43 $(39+4)$ autores de referencia y prestigio a nivel nacional. Es necesario destacar la colaboración de cuatro autores internacionales que son referentes en sus áreas a nivel mundial (Kraemer, Häkkinen, Bishop, y Maffiuletti).

La obra está organizada en cuatro secciones o bloques: 1) Análisis y observación del movimiento humano (nueve capítulos); 2) Herramientas para el entendimiento del movimiento humano (siete capítulos); 3) Aplicación práctica a la actividad física de la biomecánica (nueve capítulos); y 4) Bases neuromusculares del movimiento (once capítulos). Antes de abordar las diferentes obras de este manual, cabe destacar el hecho de que la mayoría de los capítulos tienen una extensión de entre 15 y 18 páginas, lo que hace posible su utilización como documentación de lectura para alumnos de grado y de postgrado. Además, esto permite que la lectura de este manual y de sus capítulos sea de forma no ordena$\mathrm{da}$, es decir, que el lector pueda ir leyendo los distintos capítulos del mismo sin un orden preconcebido.

Previo a los bloques de contenidos antes indicados, se incluye un capítulo de ubicación histórica, donde se abordan los antecedentes y la situación actual de esta área con el objeto de ubicar adecuadamente los contenidos que a continuación se abordan. En el primer bloque, sobre análisis y observación del movimiento humano, se diferencian a su vez dos sub-bloques: a) bases conceptuales (capítulos 2 al 5), en el cual se establecen la terminología, los conceptos, las características de la medida, y las variables de estudio propias del área. Esta información permite al lector crear el punto de partida para el resto de contenidos abordados o como recordatorio; y b) análisis y estructura del movimiento (capítulos 6 al

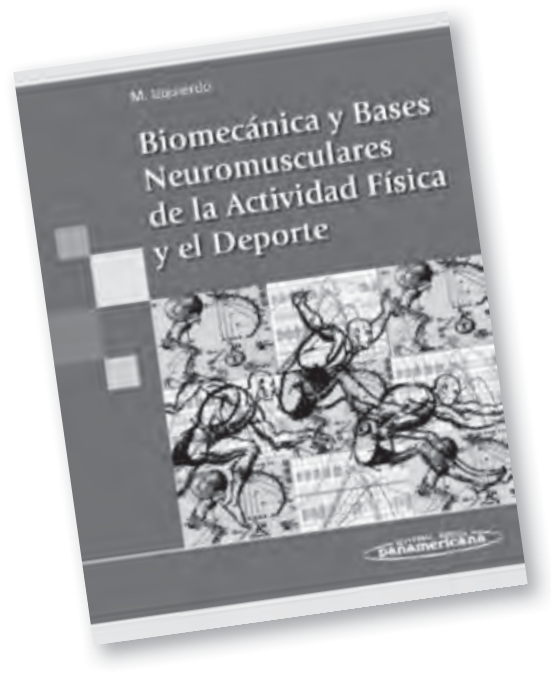

10), en el cual se aborda con gran acierto por los autores el análisis del estudio de las acciones motoras. El enfoque realizado no sólo es un enfoque sistemático y científico sino que además logra conectar el estudio mecánico del movimiento humano con el control motor, aprendizaje motor y con el estudio de las conductas humanas, y todo ello con una orientación aplicada al profesional del deporte. Este bloque finaliza con una revisión de los instrumentos y técnicas de análisis empleadas en la actividad física y el deporte.

En el segundo bloque, sobre herramientas para el entendimiento del movimiento humano, se abordan los conceptos para entender el movimiento tanto desde el estudio 
de las traslaciones y movimientos, (cinemática), como desde el punto de vista de las causas de esos movimientos, su equilibrio y estabilidad (cinética). El estudio del trabajo, potencia y energía, y de la dinámica de fluidos (agua y aire) se abordan de forma específica en sendos capítulos. Es de agradecer que todos los conceptos abordados sean explicados mediante ejemplos aplicados.

En el tercer bloque, sobre aplicación práctica a la actividad física de la biomecánica humana, se emplean las herramientas descritas en el bloque anterior para junto a los estudios científicos existentes estudiar la práctica de actividad física y deporte. Dentro de este bloque se diferencian a su vez dos subbloques: a) aplicación al rendimiento deportivo (capítulos 18 al 21), donde se aborda el estado actual del conocimiento sobre los movimientos de lanzamiento, golpeo, trayectorias y movimientos con rotación en la práctica de diferentes deportes. De forma específica, se aborda la biomecánica de la natación y el estudio de la aerodinámica del ciclismo y del atletismo; y b) aplicación al material deportivo y la prevención de lesiones (capítulos 22 al 26). Se aborda de forma monográfica el estado actual de los estudios existentes sobre el calzado deportivo y los terrenos de hierba artificial. Tras eso se profundiza en la relación entre carga, materiales y las lesiones deportivas, y cómo se puede prevenir la aparición de las mismas. Por último, se aborda la situación actual de la utilización de colchonetas en la gimnasia, sus características, sus normativas y cómo ésta puede afectar sobre la aparición de lesiones.

En el cuarto bloque, sobre bases neuromusculares del movimiento humano, se busca conocer las características del músculo y sus adaptaciones ante la realización o ausencia de ejercicio. Dentro de este bloque se diferencian a su vez dos subbloques: a) estudio de las propiedades del músculo (capítulos 27 a 31) desde la biomecánica y la fisiología (aplicación y forma de generar la tensión muscular), los protocolos para evaluar y analizar la valoración de la fuerza muscular, y la fatiga muscular (muscular y sistema nervioso) y los factores que afectan sobre ésta; y b) adaptaciones ante el envejecimiento, entrenamiento/ desentrenamiento y puesta a punto (capítulos 32 a 37). Se revisa lo que se conoce actualmente sobre la prescripción efectiva de la fuer$\mathrm{za}$, qué adaptaciones se producen en el organismo cuando este tipo de trabajo se realiza, qué adaptaciones son necesarias cuando se trabaja con atletas de rendimiento (entrenamiento, desentrenamiento y puesta a punto) y con personas de otras poblaciones.

En definitiva, se revisa desde múltiples $\mathrm{y}$ diversas perspectivas el estado actual de la biomecánica deportiva, todo ello siempre desde el enfoque científico. El manual evoluciona desde aspectos genéricos y básicos a la información más actual del estado de la investigación en biomecánica y bases neuronales. Por todo ello, sólo cabe decir que nos encontramos ante un libro de referencia sobre la temática e imprescindible en la biblioteca tanto de alumnos como de profesionales del área de Ciencias de la Actividad Física y del Deporte. 